\title{
Non Tuberculous Mycobacteria and Toxoplasma Co-Infection of the Central Nervous System in a Patient with AIDS
}

\author{
Partha Sardar ${ }^{1}$, Dipanjan Bandyopadhyay ${ }^{1}$, Deeptarka Roy ${ }^{1}$, Pradipta Guha ${ }^{1}$, Goutam Guha ${ }^{2}$, Amit Kumar Banerjee ${ }^{1}$ \\ ${ }^{1}$ Department of Medicine, Medical College; ${ }^{2}$ Department of Neurology, Medical College; Kolkata, West Bengal, India
}

\begin{abstract}
New-onset seizures are frequent manifestations in patients infected with Human Immunodeficiency Virus (HIV). We describe the clinical and radiological findings in an 25yr old AIDS patient presenting with new onset seizures as the primary manifestation of cerebral toxoplasmosis and Non Tuberculous Mycobacterial [NTM] co-infection. Cranial computed tomography showed a subtle ventricular dilatation whereas magnetic resonance imaging disclosed prominent temporal horn. Toxoplasma tachyzoites and rapidly growing mycobacteria were recovered from CSF. Seizures were complex partial in nature and refractory to antiepileptic therapy.
\end{abstract}

Key-Words: Non tuberculous Mycobacteria, toxoplasmosis, HIV, seizures.

Toxoplasmosis of the central nervous system usually presents as subacute onset focal neurological deficit and mass lesions are detected by computed tomography scanning or magnetic resonance imaging [1]. The definitive diagnosis requires direct demonstration of the tachyzoite form of Toxoplasma gondii in cerebral tissue. The presumptive diagnosis is based on serology, clinical and radiological features, and response to anti-Toxoplasma therapy [2]. Presence of CNS toxoplasma infection without mass lesion is rare. In HIV infected patients Non Tuberculous Mycobacteria [NTM] mainly causes skin and pulmonary infection. NTM specially rapid growers rarely causes CNS infection.

\section{Case Report}

A 25 year old male came into the fold of our care in May 2008, in an unconscious state along with involuntary movements and complex partial seizures involving the right upper limb and the right side of face, for the past 3 days. History revealed presence of low grade evening fever with remission during the night spread over the last 6 months. He was treated for pulmonary tuberculosis 3 years back and the ATD regimen was duly completed. He had a history of exposure to commercial sex. There was no history of weight loss, oral or genital ulcers, headache, vomiting or visual disturbances and no past history suggestive of a seizure disorder.

The general survey revealed enlarged cervical lymph nodes and hyperpigmented papules and plaques, distributed over all four limbs (Figure 1). All his vitals were within normal range. A detailed CNS examination revealed rigidity of both right upper and lower limbs. The deep reflexes were exaggerated on the right side while the plantar response was

Received on 15 May 2009; revised 6 November 2009.

Address for correspondence: Dr. Partha Sardar, MBBS. Post Graduate Trainee, Department of Medicine, Medical College Kolkata. Flat no D1, Purbasa,87 Bipplabi Ullaskar Dutta Road,Baghajatin, Kolkata 700086. West Bengal. India. E-mail: medicopartha@gmail.com. Telephone: 091-33-24256171. 091-9433905679.

The Brazilian Journal of Infectious Diseases 2009;13(6):449-451. (C) 2009 by The Brazilian Journal of Infectious Diseases and Contexto Publishing. All rights reserved. bilaterally flexor. There was no involvement of the cranial nerves, sensory or autonomic systems. The bladder and bowel control was unaffected. Ophthalmoscopy was within normal limits. Meningeal signs were positive. Abdominal exam revealed a firm nontender hepatomegaly. All other systems were apparently unaffected.

Baseline investigations documented normal haemogram, blood sugar, urea and creatinine values. The Liver Function Test showed a serum bilirubin level of $0.6 \mathrm{mg} / \mathrm{dL}$ (direct bilirubin of $0.4 \mathrm{mg} / \mathrm{dL}$ ), total protein of $8.3 \mathrm{mg} / \mathrm{dL}$ (albumin $3.7 \mathrm{mg} / \mathrm{dL}$, globulin 4.3mg/dL), SGOT 108IU, SGPT 76IU and Alkaline Phosphate $430 \mathrm{IU}$. An ultrasound of abdomen revealed enlarged liver size with normal echo-texture. He was tested positive for Anti HIV -1 antibodies (ELISA), which was confirmed by western blot. His CD4 count was 46 cells / cumm. CT and MRI scans were essentially normal except for enlarged ventricles (Figures 2 and 3). The CSF study revealed a cell count of 5 cells/ cumm (all lymphocytes). The sugar and protein level in CSF were $38 \mathrm{mg} / \mathrm{dL}$ and $84 \mathrm{mg} / \mathrm{dL}$ respectively. A smear drawn from CSF showed plenty of acid fast bacilli by ZN staining and toxoplasma tachyzoites on Giemsa stain.

We initiated CAT 2 antitubercular regimen along with pyrimethamine and co-trimoxazole. He was also started on IV phenytoin and oral oxcarbamazepine was added to his antiepileptic regimen and the dose was gradually upscaled without a fruitful outcome. Rather the complex partial seizures gradually increased in frequency along with secondary generalization.

Repeat metabolic profile and CT scan couldn't help further. A CSF study was repeated 3 weeks later which showed a cell count of 5 cells/cumm (all being lymphocytes), sugar and protein of 59mg/dL and $62 \mathrm{mg} / \mathrm{dL}$ respectively and no AFB in the CSF smear. The CSF culture done in LJ medium showed growth within 72 hrs. Subculture on MacConkey medium showed similar growth after 48 hours (Figure 4). ZN staining from growth showed acid fast bacilli (Figure 5). The EEG report was normal. Thereafter the patient was started on clarithromycin, amikacin and rifampicin. Seizures were controlled largely and the skin lesions improved but consciousness level did not. Eventually we lost the patient on the $19^{\text {th }}$ post-admission day. 
Figure 1. Hyper pigmented papules and plaques, distributed over legs.

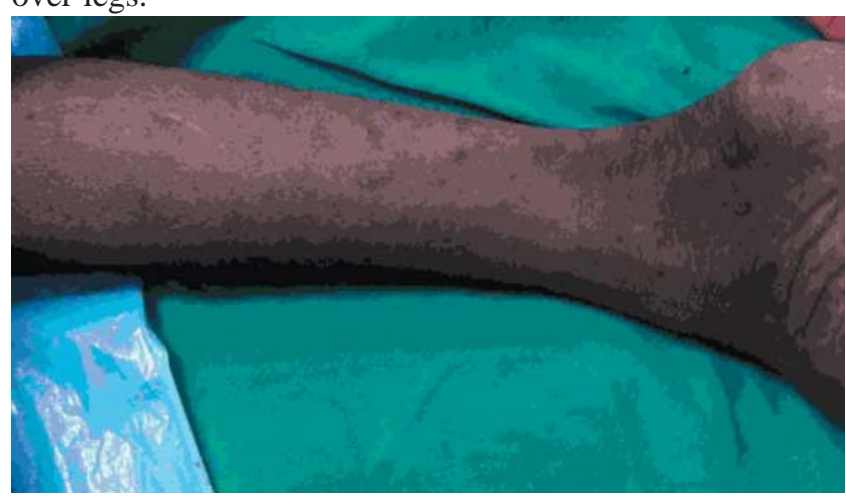

Figure 2. CT scan showing ventricular dilatation and diffuse cerebral atrophy.

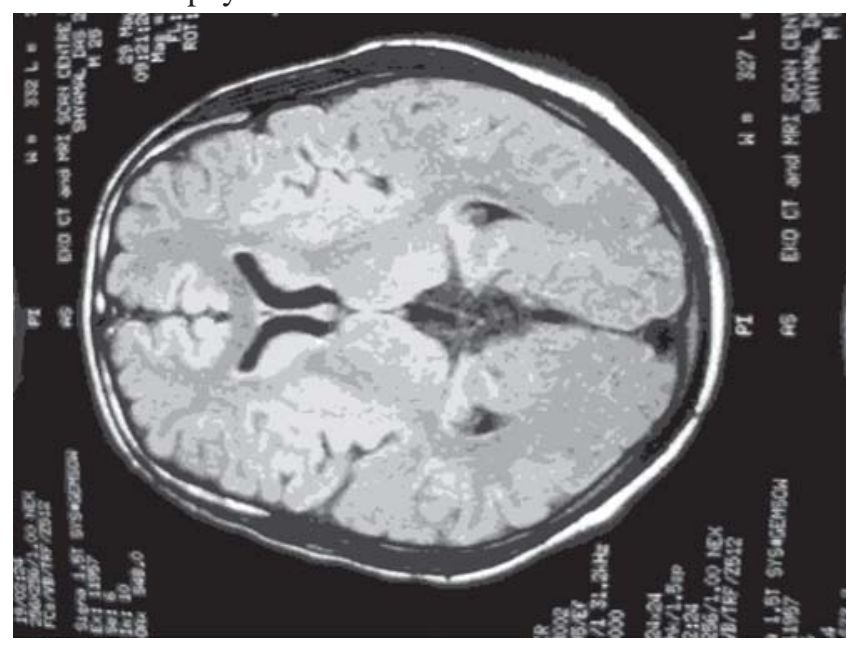

Figure 3. MRI showing prominent temporal horn.

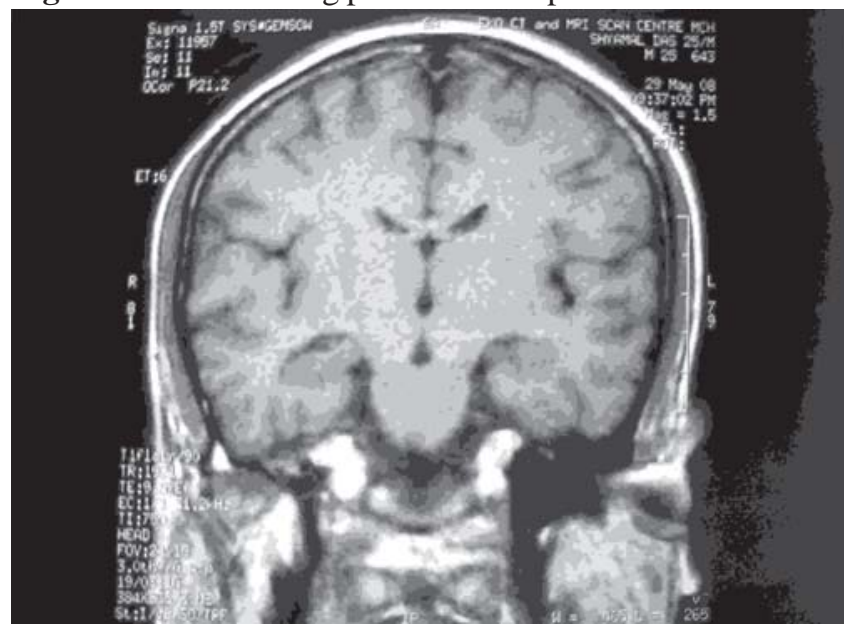

\section{Discussion}

Infection with the human immunodeficiency virus (HIV) is associated both with infections of the central nervous system and with neurological deficits arising out of the neurotropicity of the virus itself. Seizures and epilepsy are not rare among HIV-infected patients. Majority of patients with HIV infection
Figure 4. Rapid growing mycobacterial growth in LJ media.

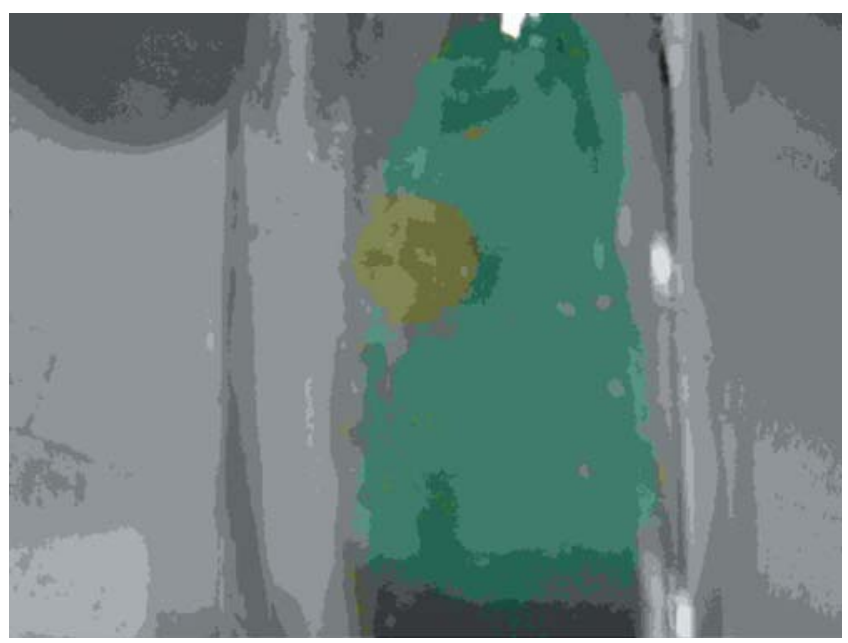

Figure 5. Acid fast bacilli with AFB stain from subculture.

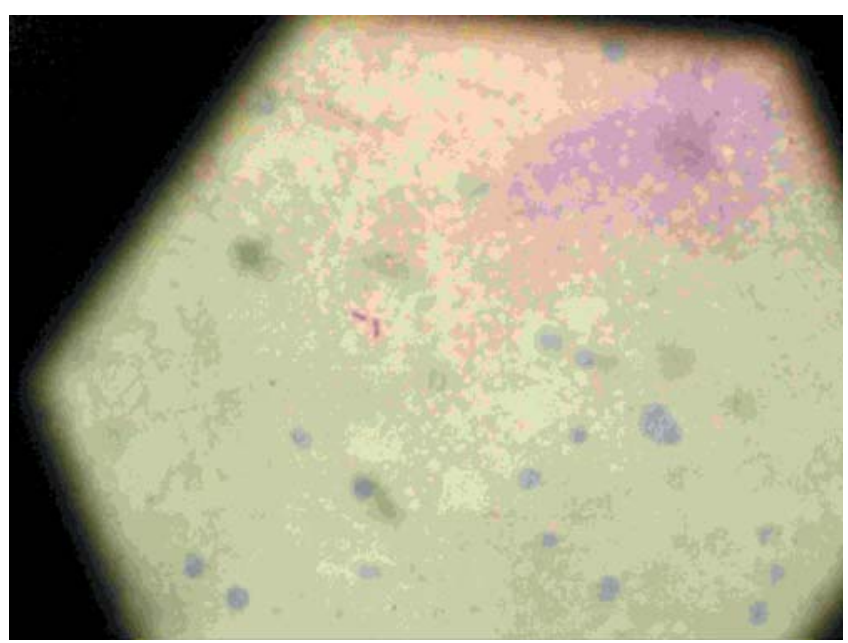

and new onset seizures have secondary brain lesion as the cause of seizure [3]. High rate of seizure recurrence mandates therapy of solitary seizure in patients with HIV infection.

The various infections associated with development of seizures in HIV infected patients include toxoplasmosis, cyrtococcosis, tuberculoma and tuberculous abscess, neurosyphilis, herpes simplex infections and nocardial abscess [3].

Toxoplasmosis is the most frequent cause of intracranial mass lesions in patients with AIDS, accounting for 50-70\% of all mass lesions in this population Clinical presentation of CNS toxoplasmosis ranges from headache and fever without neurological symptoms to coma. Seizures as the only presentation is rare [3]. Multiple small, uniformly ring enhancing lesions with moderate to marked surrounding edema in the basal ganglia and subcortical regions suggests the diagnosis. Ventriculitis and hydrocephalus without any 
focal parenchymal lesion may be the only manifestations of CNS toxoplasmosis. Sell M and Klingebiel R reported a case of Primary cerebral toxoplasmosis presented with ventriculitis and hydrocephalus in an AIDS patient [4]. It is important to recognize this unusual form of presentation of cerebral toxoplasmosis in order to perform specific therapy.

There is high prevalence of tuberculosis in patients with HIV infection; hence the role of non-tuberculous mycobacteria (NTM) in HIV patients has always been undermined. NTM may be responsible for clinical disease in a substantial number of immuno-compromised HIV sero-positive individuals even in a country endemic for Mycobacterium tuberculosis ( $M$. tuberculosis). Rapid growing mycobacteria have usually been associated with disseminated skin and soft tissue infections and pulmonary infections [5].Meningitis caused by rapidly growing subgroups of atypical mycobacteria is largely unheard of. In the present case atypical mycobacterial infection (rapid growers) and CNS toxoplasmosis possibly were the underlying cause of refractory seizures. Despite the high endemicity of tuberculosis in developing countries, the presence of NTM should be ruled out; especially in immunocompromised HIV seropositive individuals before instituting anti-tubercular therapy empirically. In addition, non-response of NTM to ATD may be wrongly attributed to multi-drug resistant tuberculosis. Speciating our case, we need to keep some important points in mind. The three most important clinical pathogenic species that represent more than $80 \%$ of clinical isolates of Rapidly Growing Mycobacteria include $M$. fortuitum, M. chelonae and M. abscessus. Only a very small number of cases of CNS infections due to Rapidly Growing Mycobacteria have been documented, majority of these cases are related to infections with $\mathrm{M}$. fortuitum. $\mathrm{M}$. fortuitum can also be rarely associated with disseminated skin lesions [6]. We are reporting this case because CNS involvement with toxoplasma and rapid grower mycobacterial coinfection was never reported before. CNS toxoplasma presenting solely as refractory seizures with CT/MRI features of ventriculitis is also very rare.

\section{References}

1. Caramello P, Forno B, Lucchini A et al. Meningoencephalitis caused by Toxoplasma gondii diagnosed by isolation from cerebrospinal fluid in an HIV-positive patient. Scand J Infect Dis. 1993;25(5):663-6.

2. José E. Vidal, Anne Spichler, Augusto C. P. de Oliveira and André Villela Lomar; Meningoencephalitis and New Onset of Seizures in a Patient with Normal Brain CT and Multiple Lesions on MRI:The Brazilian Journal of Infectious Diseases 2004;8(1):115117.

3. Garg RK. HIV infection and seizures: Postgrad Med J. 1999;75(885):387- 90.

4. Sell M, Klingebiel R, Di Iorio G, Sampaolo S. Primary cerebral toxoplasmosis: a rare case of ventriculitis and hydrocephalus in AIDS. Clin Neuropathol. 2005;24(3):106-11.

5. Phowthongkum P, Prasanthai V, Udomsantisook N, Suankratay C; Rapidly growing mycobacteria in King Chulalongkorn Memorial Hospital and review of the literature in Thailand: J Med Assoc Thai. 2005;88(8):1153-62.

6. David Schlossberg. Tuberculosis and Nontuberculous Mycobacterial Infections, Fifth Edition. Tata McGraw-Hill Edition 2007. 